\title{
A computational lifetime prediction of a thermal shock experiment. Part I : thermomechanical modelling and lifetime prediction
}

\author{
S. AMIABLE ${ }^{1}$, S. CHAPULIOT ${ }^{2}$, A. CONSTANTINESCU ${ }^{1}$ and A. FISSOLO ${ }^{2}$ \\ ${ }^{1}$ Solid Mechanics Laboratory - CNRS UMR 7649, Department of Mechanics - Ecole Polytechnique, 91128 Palaiseau cedex, France, ${ }^{2}$ DM2S/SEMT, \\ CEA Saclay, 91191 Gif sur Yvette cedex, France
}

\begin{abstract}
The SPLASH experiment has been designed in 1985 by the CEA to simulate thermal fatigue due to short cooling shocks on steel specimens and is similar to the device reported by Marsh in Ref. [1]. The purpose of this paper is to discuss the mechanical and the fatigue analysis of the experiment using results from FEM computations. The lifetime predictions are obtained using a modified dissipated energy with a maximal pressure term and agree with the experimental observations. The numerical analysis of the mechanical state shows an important evolution of the triaxiality ratio during the loading cycle. Further comparisons and discussions of the fatigue criteria are provided in the second part of the paper (Part II) ${ }^{2}$.
\end{abstract}

Keywords 304 stainless steel; finite element; low-cycle fatigue; plasticity; thermal fatigue; thermal shock.

\section{NOMENCLATURE}

$\mathbf{A}=$ elastic 4th order tensor

$c, k=$ thermal capacity and conductivity

$E, v=$ young's modulus and Poisson's coefficient

$\mathcal{F}_{2}=$ second invariant of the deviatoric stress tensor

$P=$ hydrostatic pressure

$p=$ cumulated plastic strain

$q=$ thermal flux

$r=$ heat source

$T=$ temperature field

$T F=$ triaxiality factor

$\mathbf{u}=$ displacement field

$W_{p}=$ dissipated energy density per cycle

$\Delta \sigma, \Delta \epsilon=$ equivalent stress and strain ranges

$\boldsymbol{\epsilon}, \mathbf{e}=$ strain tensor and its deviatoric part

$\epsilon^{e}, \epsilon^{p}=$ elastic and plastic strain tensors

$\rho=$ volumic mass

$\sigma_{Y}=$ elastic limit

$\boldsymbol{\sigma}, \mathbf{s}=$ stress tensor and its deviatoric part

\section{INTRODUCTION}

A series of industrial structures like turbines, engines, nuclear or classical thermal plants are subject to thermomechanical cycling leading to fatigue. The actual design and

Correspondence: A. Constantinescu. E-mail: andrei.constantinescu@lms. polytechnique.fr assessment methods are generally based on design rules provided by different codes (see for example Ref. [3]). These rules stem from closed-form solutions and experimental studies of the last decades and as such are not always appropriate to design, for example when the stress path is multiaxial during the cycle.

The finite element method provides a powerful tool for the mechanical analysis of structures, but its success 
is determined by a series of factors: correct constitutive law and boundary conditions, computational burden. Moreover the results present often multiaxial stress-strain paths which are difficult to interpret with respect to classical criteria defined in uniaxial context, and therefore further work is needed to adapt the fatigue criterion for these cases.

The purpose of this work is to provide a fatigue analysis of the SPLASH test rig. The experiment imposes thermal shocks to a heated specimen by spraying cold water on it and leads under certain conditions to crack initiation. Certain features of the experiment are important spatial and temporal gradients of the mechanical fields involved. The originality of this work is two-fold. On the one hand, it adapts and interprets classical numerical techniques to obtain a precise thermomechanical analysis of the SPLASH experiment. On the other, it provides a novel discussion of low cycle thermomechanical fatigue criteria involving a varying triaxiality ratio during the loading cycle. The numerical method is presented in this paper (Part I) and continues with the discussion of fatigue criteria in the second part (Part II). ${ }^{2}$

Regarding previous studies on structures, we might mention the studies on cast-iron exhaust manifolds and aluminum cylinder heads ${ }^{4,5}$ where damage was supposed to be uncoupled from the constitutive behaviour. A dissipated energy criterion has been used for lifetime predictions based on the mechanical fields computed on the plastic shakedown cycle. For exhaust manifolds and cylinder heads, the effect of hydrostatic pressure could be neglected. However, this is not the case for the present work and we discuss this point in the next sections and in the second part (Part II) of the paper. Other studies ${ }^{6}$ proposed to couple the constitutive law with damage in order to estimate the lifetime of crossed specimen in a biaxial straincontrolled experiment and the authors obtained comparable results from coupled and uncoupled models. This method has its computational drawbacks as it imposes to compute all cycles.

This paper presents in the first section a description of the SPLASH experiment. It continues with the description of the used $304 \mathrm{~L}$ stainless steel and the main assumptions of the modelling. The FEM analysis and the computational results are presented in section 'The thermomechamical analysis'. The last section discusses the lifetime predictions using a criterion involving dissipated energy density of the cycle and maximal pressure.

\section{THE SPLASH EXPERIMENT}

The SPLASH experiment is a thermomechanical fatigue experiment designed by the CEA in order to induce crack networks by a cool-down shock. A series of results regarding fatigue experiments have already been reported in Ref. [7] on a 316L stainless steel and in Ref. [8] for 304L stainless steel.

The basic concept of the experimental rig is similar to the one reported by Marsh in Ref. [1]. During the test, a parallepipedic specimen $\left(200 \times 30 \times 20 \mathrm{~mm}^{3}\right)$ is continuously heated by Joule effect at the maximum temperature $T_{\max }$ and then cyclically cooled by water sprays on small spots (approximatively $10 \times 5 \mathrm{~mm}^{2}$ ) on two opposite faces. At the end of the water spraying, the minimal temperature $T_{\min }$ is reached in the locally quenched zones. A photo and a schematic plot of the SPLASH test facility are represented in Fig. 1.

The temperature variation characterizing the test is defined as the difference between maximal and minimal temperature, $T_{\max }$ and $T_{\min }$, respectively, at the centre of the quenched zone:

$\Delta T=T_{\max }-T_{\min }$

The control of the temperature in the quenched zone and in-depth different points has been measured with thermocouples on a mock specimen.

The cycle starts from a homogeneous maximal temperature $T_{\max }$ in the specimen. On local zones on two opposite faces, the cooling period of $0.25 \mathrm{~s}$ to the minimal temperature $T_{\min }$ is caused by the water spray. Finally, the cycle continues with the heating up period of $7.5 \mathrm{~s}$ to $T_{\max }$ by Joule effect. During the heating, the maximal temperature $T_{\max }$ is uniformly reached over the specimen.

Due to the short cooling period and the localized spatial distribution of temperatures, the experimental device is capable of reproducing high temperature gradients. The cooling rate is of approximatively $\approx 600{ }^{\circ} \mathrm{Cs}^{-1}$ and the spatial gradient reaches about $\approx 100{ }^{\circ} \mathrm{Cmm}^{-1}$. However the affected zone is confined to some square millimeters at the surface and to about two millimeters in depth.

The experiment can be assimilated to a local quenching of the specimens and leads to a small localized plastic zone. After about 100000 cycles, an evolving crack network appears. The evolution of the crack network has been determined by optical microscopy at regular time intervals and an extended report is available in Ref. [8].

\section{MATERIAL PROPERTIES AND MODELLING ASSUMPTIONS}

The steel studied here is a $304 \mathrm{~L}$ type austenitic stainless steel with standard material composition as displayed in Table 1. Some classical elastoplastic and thermal properties in the considered temperature range of $20-300^{\circ} \mathrm{C}$ are given in Table 2 . We shall not mention viscosity as it is generally accepted that creep at this temperature can be neglected for this steel (see the constitutive law in section 'The mechanical analysis'). 
Fig. 1 SPLASH specimen.
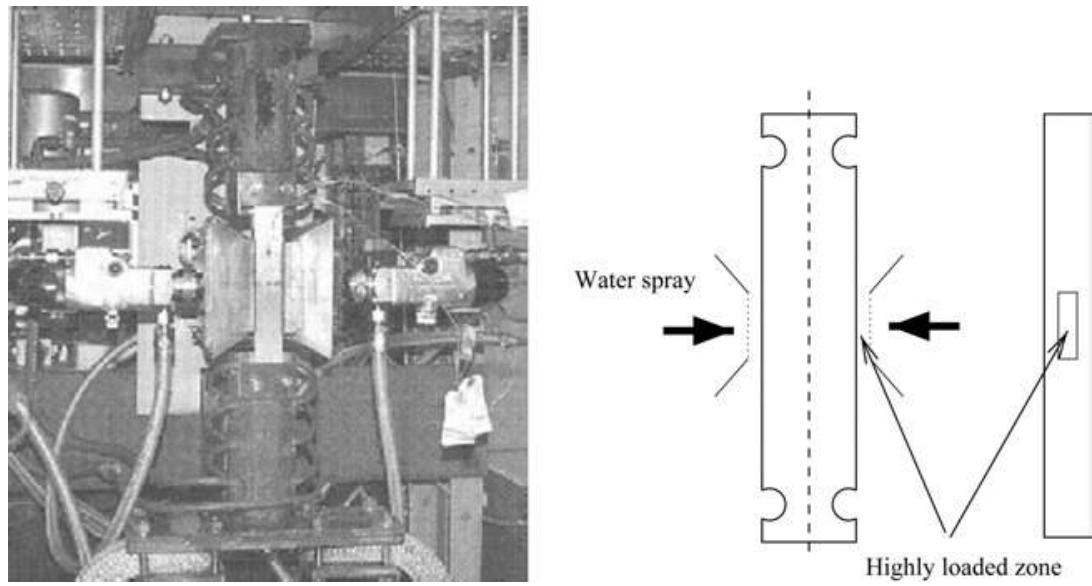

Fig. 2 Evolution of the global load in isothermal uniaxial experiment under fixed

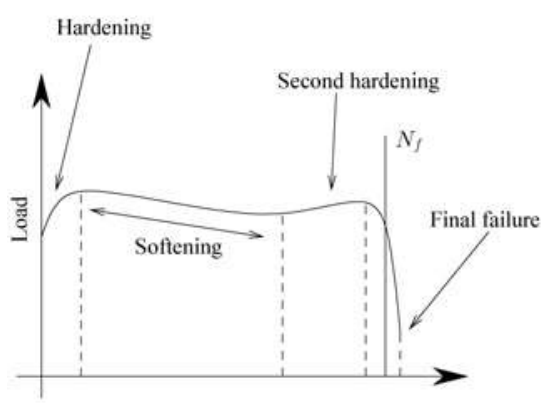

Microscopic crack initiation

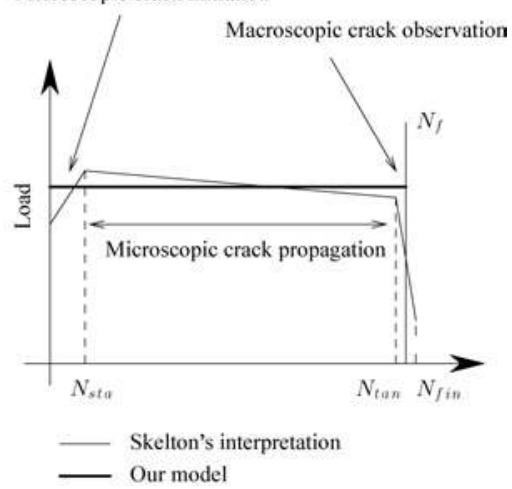
strain range.

Table 1 Chemical composition (wt\%), 304L stainless steel

\begin{tabular}{ccccccccccccccc}
\hline & $\mathrm{C}$ & $\mathrm{Mn}$ & $\mathrm{Si}$ & $\mathrm{Cr}$ & $\mathrm{Ni}$ & $\mathrm{Mo}$ & $\mathrm{S}$ & $\mathrm{P}$ & $\mathrm{Cu}$ & $\mathrm{Al}$ & $\mathrm{B}$ & $\mathrm{N}$ & $\mathrm{Fe}$ \\
\hline 304 & 0.031 & 1.48 & 0.55 & 19.4 & 8.6 & 0.23 & 0.003 & 0.028 & 0.17 & 0.025 & 0.0015 & 0.058 & Bal. \\
\hline
\end{tabular}

Table 2 Material properties, 304L stainless steel

\begin{tabular}{lcccccc}
\hline & $E(\mathrm{GPa})$ & $\alpha\left(\mathrm{K}^{-1}\right)$ & $v$ & $\rho\left(\mathrm{Kg} / \mathrm{m}^{3}\right)$ & $k(\mathrm{~W} / \mathrm{m} / \mathrm{K})$ & $C_{P}(\mathcal{7} / \mathrm{Kg} / \mathrm{K})$ \\
\hline $20^{\circ} \mathrm{C}$ & 196 & $15.910^{-6}$ & 0.3 & 7800 & 18 & 550 \\
$350^{\circ} \mathrm{C}$ & 172 & $17.810^{-6}$ & 0.3 & - & - & - \\
\hline
\end{tabular}

304L stainless steel has an impressive ductility in fatigue experiments. In isothermal uniaxial fatigue experiments in Ref. [9], it can withstand up to $10^{4}-10^{5}$ cycles under an imposed strain amplitude $\Delta \epsilon \approx 0.4-1.0 \%$. The general evolution of the load in these experiments expressing the cyclic material behaviour follows a pattern as the one sketched in the left panel of Fig. 2. The choice of a complex nonlinear constitutive law with both isotropic and kinematic hardening as the one described in Ref. [10,11,12] is necessary to represent correctly the cyclic behaviour of 304L stainless steel. However, this would conduct to heavy numerical burden because we should compute all the cycles to the final failure.

In this study, we assume that the material reaches within few cycles a stabilized cycle, i.e a plastic shakedown state. Moreover, we further suppose that this cycle will not be modified during the complete lifetime of the structure which eliminates an isotropic hardening component in the constitutive law. The final choice will, therefore, be a nonlinear kinematic hardening (see section 'The 
mechanical analysis'). The assumption of a stabilized cycle during the lifetime leads to an approximate error of about $5 \%$ in the computed stress level. We recall that these assumptions are consistent with the interpretations given by Skelton ${ }^{13}$ of the cyclic behaviour of the material and its fatigue damage under anisothermal conditions. The steps of the cyclic material behaviour defined by Skelton are delimited by the stabilization, the tangent and the final points (right panel of Fig. 2). The mechanical response at $N_{f} / 2$ lies within the stabilized period and will correspond to the material behaviour identified for computational needs.

The thermomechanical cycles of the SPLASH specimen start from a homogeneous heated specimen followed by a spray cooling period and a heating up to the initial homogeneous state.

The stabilized thermomechanical state of the specimen is determined from two uncoupled computations: a thermal analysis and a mechanical analysis with the previous computed temperature field as a given loading parameter. The uncoupling hypothesis is justified by the negligible thermal heating due to the inelastic deformation compared to the global heating problem.

The constitutive law has been considered to be uncoupled from the damage evolution. This assumption has been already justified in a series of papers. ${ }^{6,14,4,15} \mathrm{We}$ shortly recall here that the fatigue analysis will be based on a stabilized cyclic behaviour, i.e. a plastic shakedown state, which has to be obtained as the result of the elastoplastic analysis.

\section{THE THERMOMECHANICAL ANALYSIS}

All computations have been performed using the objectoriented finite element code cast $3 \mathrm{M}^{16}$ and are described in detail in the following paragraphs. The same mesh

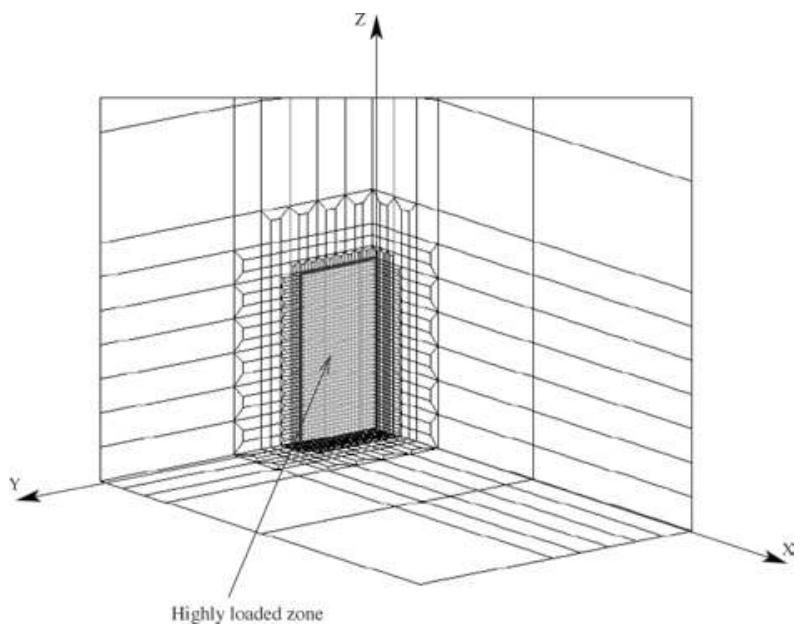

Fig. 3 SPLASH specimen mesh. has been used for thermal and mechanical computations and is represented in Fig. 3. The finest zone of the mesh corresponds to the quenched part of the specimen. The displayed mesh had 17000 nodes and combined linear cubical and prismatic elements. Different meshes have been tested in order to estimate the possible error caused by the large spatial gradients. Mesh refining beyond the limit defined in the described mesh shows to give similar results with an important increase of the computing time.

The computations have been performed on a $3 \mathrm{GHz}$ biprocessor with 4GO RAM running under Linux. A load increment for the thermal computations takes about $10 \mathrm{~s}$ while the increment for the elastoplastic computations takes about $180 \mathrm{~s}$.

\section{The thermal analysis}

The thermal problem is governed by the heat equation for the temperature field $T$ :

$\rho c \frac{\partial T}{\partial t}-\operatorname{div} k \nabla T=r$,

where $c, k$ are the heat capacity and the conductivity of the material, respectively (given in Table 2 for the $304 \mathrm{~L}$ studied here). $r$ denotes the heat source given by the Joule effect $\left(r \approx 9.10^{5} \mathrm{Wm}^{-3}\right)$. It has been identified in order to match the measured temperatures from a series of thermocouples installed on a mock specimen.

All boundary conditions have been modelled by equivalent thermal fluxes:

$-\left.k \frac{\partial T}{\partial n}\right|_{S}=q$.

The flux on the part of the specimen in direct contact with air has been considered as constant and estimated from convection formulas as $\approx 5.10^{3} \mathrm{Wm}^{-2}$. The cooling of the water spray has been considered as a flux of the following elliptical spatial distribution:

$q(y, z)=\frac{q}{2^{2 \mathrm{n}}}\left(1+\cos \frac{\pi y}{l}\right)^{n}\left(1+\cos \frac{\pi z}{b}\right)^{n}$.

This flux was considered as constant during the water spraying and absent otherwise. The amplitude $q$ of the flux has been identified to be $\approx 2.10^{6} \mathrm{Wm}^{-2}$ and the power coefficient $n$ has been considered equal to 0.2 in order to match experimental temperature measurements. After computing up to three cycles, we can report that the temperature cycle is almost stabilized after the first cycle.

In Fig. 4, we have displayed a comparison between the computed and measured temperature by a thermocouple in the centre of the quenched zone. We remark the huge temperature rate during a very short period creating 
important gradients in the specimen. As a consequence, we have needed 80 time steps in order to represent correctly the evolution of the temperature.

The temperature distribution at the surface of the quenched zone has a bell shape as displayed in Fig. 5. Its values at the limit of the quenched zone do not change during the experiment proving again the confined character of the phenomena.

\section{The mechanical analysis}

The mechanical response of the specimen to the thermal loading is determined by solving the balance equation:

$\operatorname{div} \sigma=0$

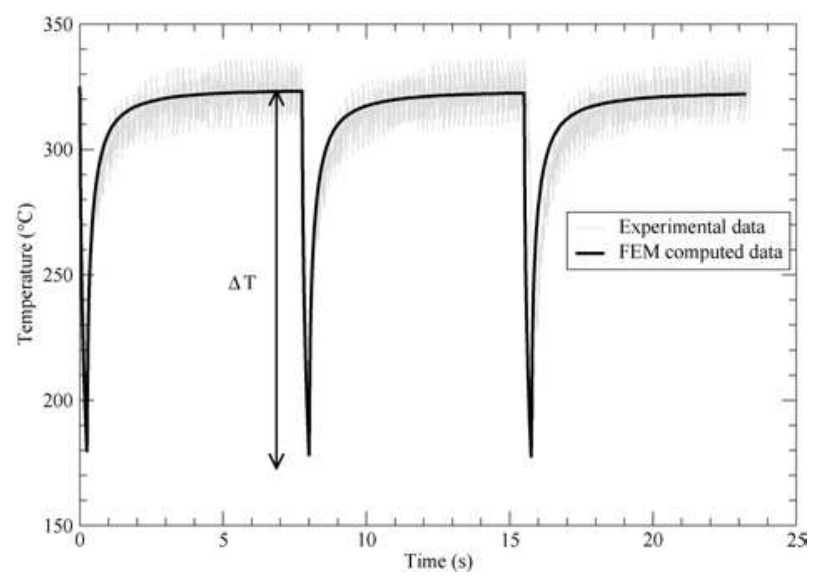

Fig. 4 Evolution of temperature with time during the first three cycles at center of the quenched zone for $\Delta T=150^{\circ} \mathrm{C}$. using an elastoplastic constitutive law, the relation between the tensors of strain $\epsilon$ and stress $\sigma$, under the assumption of small strains:

$\epsilon=\frac{1}{2}\left(\nabla+\nabla^{T}\right) u$

The modelling assumptions mentioned in the section 'Material properties and modelling assumptions' lead to a constitutive law without taking into account viscosity effects and without isotropic hardening. Therefore, we have chosen an elastoplastic constitutive law based on a classical additive decomposition of the strain into an elastic and a plastic part, a Von Mises yield criterion $\mathcal{f}_{2}$ and a nonlinear kinematic hardening rule, as shown in the following equations:

$\epsilon=\epsilon^{e}+\epsilon^{p}$

$\boldsymbol{\sigma}=\mathbf{A}: \boldsymbol{\epsilon}^{e}=\mathbf{A}:\left(\boldsymbol{\epsilon}-\boldsymbol{\epsilon}^{p}\right)$

$\dot{\boldsymbol{\epsilon}}^{p}=\dot{\lambda} \frac{\partial f}{\partial \boldsymbol{\sigma}}$

$f=\sqrt{3 \mathcal{F}_{2}(\boldsymbol{\sigma}-\mathbf{X})}-\sigma_{Y}$

$\dot{\mathbf{X}}=b\left(\frac{2}{3} g \dot{\boldsymbol{\epsilon}}^{p}-\mathbf{X} \dot{p}\right)$

$\dot{p}=\sqrt{\frac{2}{3} \dot{\epsilon}^{p}: \dot{\boldsymbol{\epsilon}}^{p}}$

This constitutive law is completely determined by four materials parameters: the Young's modulus $E$, the elastic limit $\sigma_{Y}$ and two hardening coefficients, $g$ and $h$.
Fig. 5 Spatial distribution of the temperature at the end of the cooling period for $\Delta T=150^{\circ} \mathrm{C}$
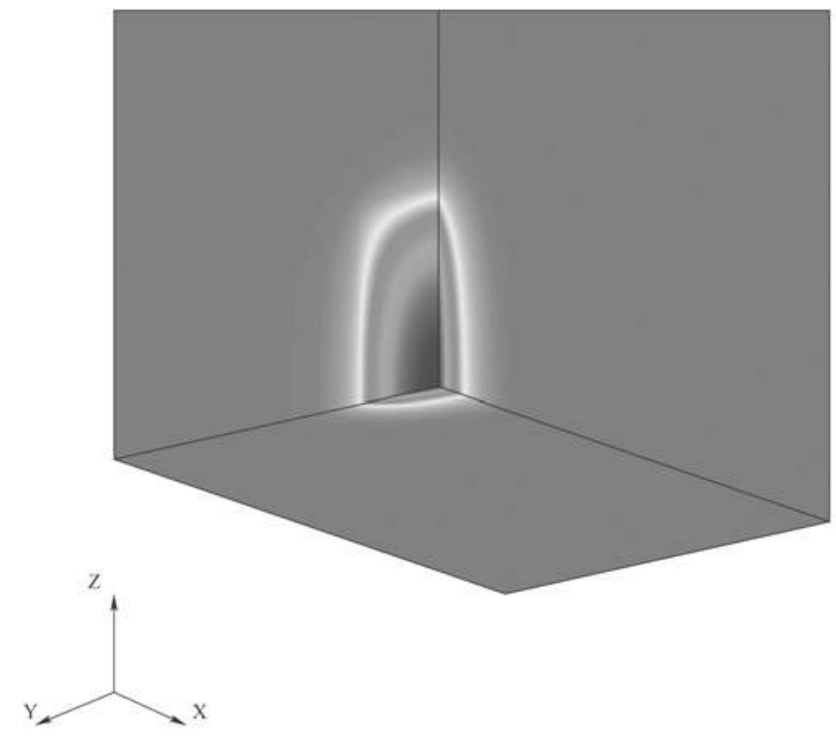
These parameters have been identified by minimizing the merit function:

$\frac{1}{2} \int\left\|\sigma^{c}-\sigma^{e}\right\|^{2} d t$,

where $\boldsymbol{\sigma}^{c}$ is the computed stress and $\boldsymbol{\sigma}^{e}$ is the experimentally measured stress from the cycle at $N_{\mathrm{f}} / 2$. The experiment was an uniaxial isothermal LCF test conducted at $320^{\circ} \mathrm{C}$ under imposed strain range. ${ }^{9}$

Differences with measurements at $165{ }^{\circ} \mathrm{C}$ have been proven to be negligible and therefore the variation of material parameters with temperature has been neglected in this work as the temperature range of the SPLASH experiment lies between $165-320^{\circ} \mathrm{C}$.

The identification procedure is based on a LevenbergMarquart minimization algorithm (from Mathematica ${ }^{17}$ ) and a gradient computed using finite differences based on direct computations with Cast $3 \mathrm{M} .{ }^{16}$ Without discussing further details of the identification procedure here, let us just observe the match between the computed and experimental material response as displayed in Fig. 6. The identified parameters (Table 3) were in good agreement with the orders of magnitudes reported in literature. ${ }^{10}$

The computed configurations correspond to three different imposed temperature differences $\Delta T$ at the surface of the specimen (Eq. 1). In each case, up to 30 cycles have been computed in order to ensure that the plastic shakedown has been attained and that the mechanical stabilized cycle is correctly predicted. However, only 3-5 cycles were generally sufficient to obtain the stabilized mechanical behaviour.

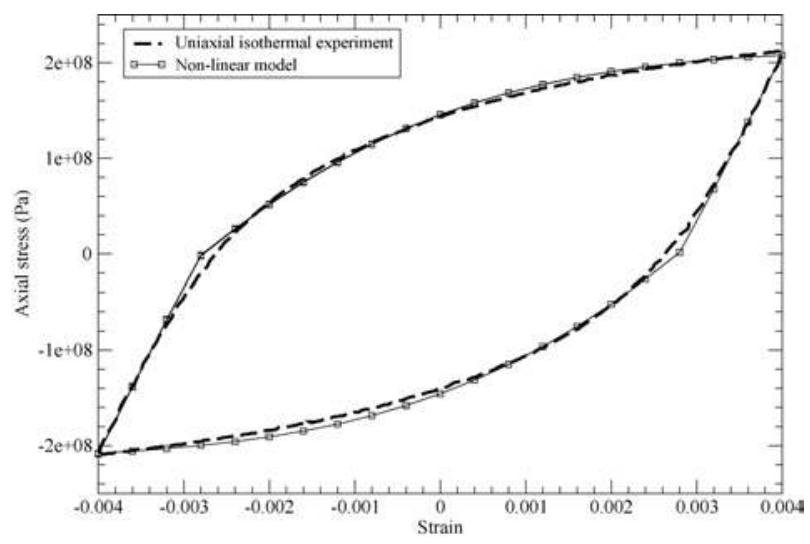

Fig. 6 Identification of constitutive law on isothermal uniaxial LCF test at $320^{\circ} \mathrm{C}$.

Table 3 Materials parameters identified at $320^{\circ} \mathrm{C}$

\begin{tabular}{lcccc}
\hline$E(\mathrm{GPa})$ & $v$ & $\sigma_{Y}(\mathrm{MPa})$ & $g(\mathrm{MPa})$ & $b$ \\
\hline 175 & 0.3 & 102 & 114 & 532 \\
\hline
\end{tabular}

We define the equivalent plastic strain $\epsilon_{\mathrm{eq}}^{\mathrm{p}}$ as a scalar quantity computed by:

$\epsilon_{\mathrm{eq}}^{\mathrm{p}}=\sqrt{\frac{2}{3} \epsilon^{p}: \epsilon^{p}}$

The bell shape of the computed distribution of equivalent plastic strain is displayed in Fig. 7 and is similar for all imposed temperature differences. Its evolution with depth is given in Fig. 8. We remark that the temperature difference $\Delta T$ influences the values of the plastic strain at the surface, but it does not influence the plastic penetration depth. The accuracy of this result has been checked with respect to the mesh size of the finite element computations and no variations have been observed.

The evolution of strain versus stress components exhibits classical loops as displayed in Fig. 9. The values of stresses suggest that the stress state is multiaxial. If we define the triaxiality factor $T F$ at each time instant as:

$$
\begin{aligned}
T F & =\frac{P}{\sqrt{3 \mathcal{F}_{2}(\boldsymbol{\sigma})}} \\
P & =\frac{1}{3} \operatorname{Tr}(\boldsymbol{\sigma}) \quad \mathcal{F}_{2}(\boldsymbol{\sigma})=\frac{1}{2} \mathbf{s}: \mathbf{s} \quad \mathbf{s}=\boldsymbol{\sigma}-P \cdot \mathbf{I}
\end{aligned}
$$

and display its values versus the temperature at the centre of the quenched zone (see Fig. 10), we remark an important evolution of $T F$ during the cycle, from -0.75 to 1.40 .

\section{THE FATIGUE ANALYSIS}

In this section, we provide a fatigue analysis with respect to a proposed fatigue criterion combining the dissipated energy per cycle and the maximum hydrostatic pressure. The number of cycles to failure $N_{\mathrm{f}}$ in the SPLASH experiment, corresponds to the initiation of a surface crack with a length of $100 \mu \mathrm{m}$. Further explanations for the choice of this criterion and the definition of $N_{\mathrm{f}}$ are given in the second part of this paper (Part II). We shall only mention here that this multiaxial criterion takes into account both deviatoric and hydrostatic parts of the stress tensor, as well as the plastic strain.

The fatigue criterion understood as a crack initiation criterion is classically defined as a relation between a local function $\Phi$ computed in each point $x$ of the structure $\Omega$ and the number of cycles to failure of the structure:

$\max _{x \in \Omega}\left[\Phi\left(\epsilon, \epsilon^{p}, \sigma, \ldots\right)\right]=a N_{\mathrm{f}}^{b}$,

where $a, b$ are two material parameters and $\epsilon, \epsilon^{p}, \sigma$ are the computed values of the fields during the stabilized cycle. It assumes implicitly that the structure reaches a stabilized condition without any ratcheting effect. This assumption has been verified in the previous section, but we shall 
Fig. 7 Equivalent plastic strain $\epsilon_{\mathrm{eq}}^{p}$ at the end of the cooling period for $\Delta T=150^{\circ} \mathrm{C}$.

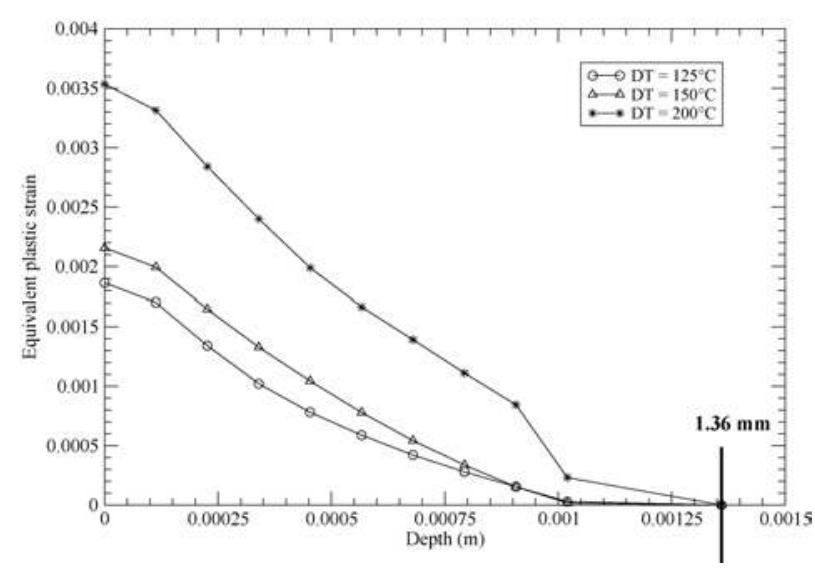

Fig. 8 Evolution of the equivalent plastic strain $\epsilon_{\text {eq }}^{p}$ with depth at the centre of the quenched zone at the end of the cooling period.

understand that it is a direct consequence of the initial choice of the constitutive law.

The fatigue parameter $\Phi$ proposed here is defined as:

$\Phi=W_{p}+\alpha P_{\max }$

where $\alpha$ is a material constant, $W_{p}$ is the dissipated energy density per cycle and $P_{\max }$ is the maximal hydrostatic pressure per cycle:

$W_{p}=\int_{\text {cycle }} \boldsymbol{\sigma}: \dot{\boldsymbol{\epsilon}}^{p} d t$

$P_{\max }=\max _{t} P(t)$.

The form of this fatigue parameter is close to the fatigue criteria of Sines, Crossland or Dang Van used in the high cycle fatigue regime, ${ }^{18}$ where the function $\Phi$ is splitted

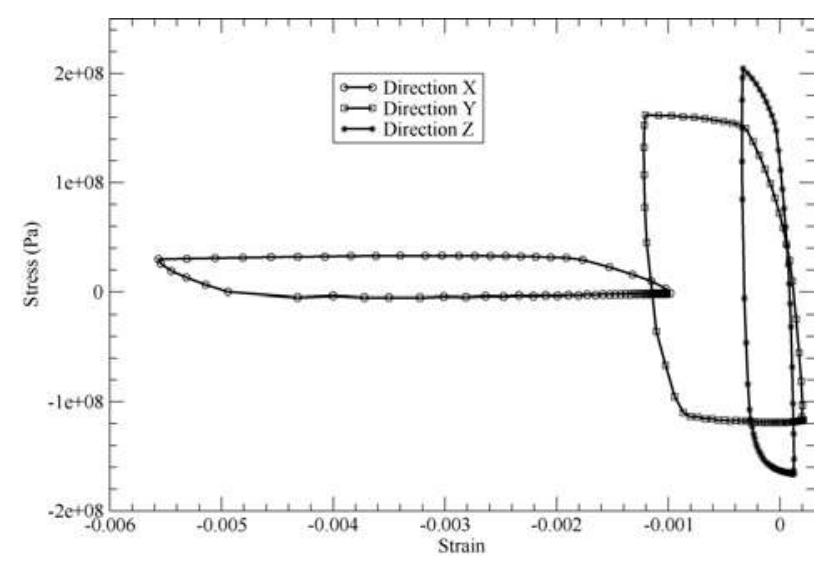

Fig. 9 Evolution of the strain versus stress components during the stabilized cycle for $\Delta T=150^{\circ} \mathrm{C}$.

additively between a deviatoric norm and a spherical norm of the stress path, for example $\mathcal{f}_{2}$ and respectively $P_{\mathrm{m}}$ or $P_{\max }$.

Figure 11 compares the predicted versus experimental lifetimes for the SPLASH experiment and for uniaxial and multiaxial tests from the literature. ${ }^{9,19}$ One can remark that the SPLASH experiments are well estimated, as the points lie between the \pm 2 lifetimes of the medium line.

\section{CONCLUSION}

This paper presented the analysis of the SPLASH thermal shock test rig. The analysis provides a complete lifetime prediction method based on a thermomechanical finite element computation of the experiment. The fatigue failure has been estimated using a proposed a posteriori criterion 


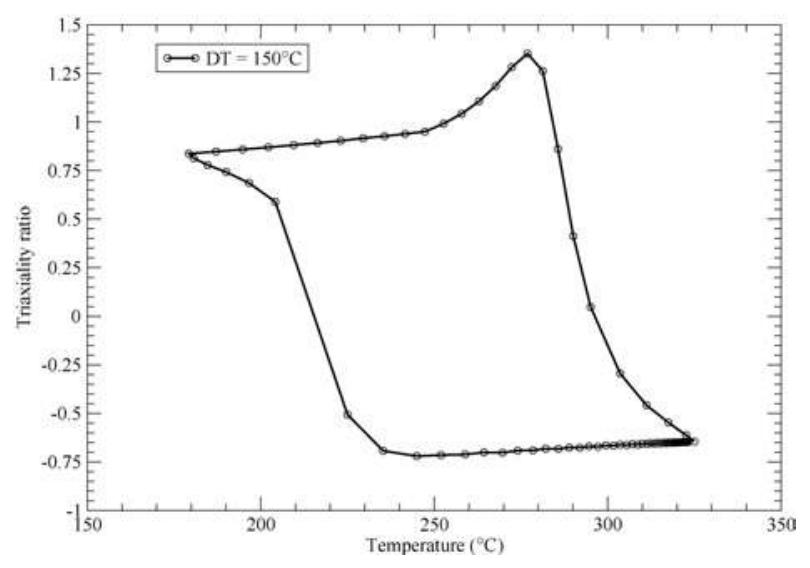

Fig. 10 Evolution of triaxiality during the stabilized cycle for the SPLASH test at $\Delta T=150^{\circ} \mathrm{C}$

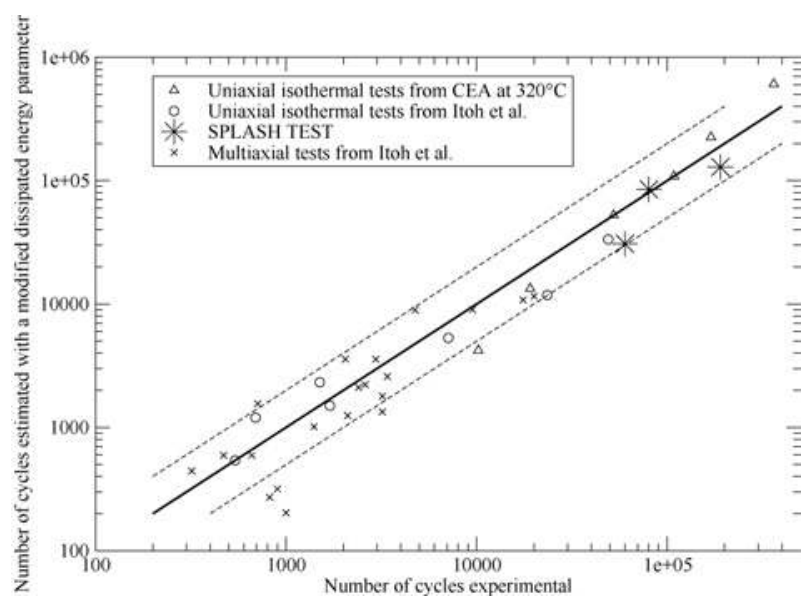

Fig. 11 Comparison of experimental vs. predicted lifetime for various experiments and the complete elastoplastic analysis for SPLASH using the modified energy fatigue parameter. The experiments are : uniaxial isothermal tests from $\mathrm{CEA}^{8}$ at $320^{\circ} \mathrm{C}$, different multiaxial and uniaxial experiments from literature ${ }^{18}$ at $25^{\circ} \mathrm{C}$

based on the dissipated energy density and the maximum hydrostatic pressure per cycle.

The analysis proved that precise fully three-dimensional finite element computations can be done in spite of the computational burden imposed by the high temporal and spatial gradients. The results showed an important evolution of the triaxiality factor during the loading cycle which can not be estimated using closed form solutions or simplified models currently used for this experiment in Ref. [3].

The lifetime prediction matches the fatigue results from the SPLASH experiments and from other tests in the literature, indicating the robustness of the proposed approach. The complete computational chain provides, therefore, a method for structural design.
The modified energy criterion takes into account the effect of triaxiality and permits a good match of the experimental data. However, it does not provide an understanding of the underlying damage phenomena, at a smaller scale. To do this, a better understanding of the global dissipation phenomena, as well as the local initiation and propagation of the crack network should be reached. As such, we would expect that a fatigue criterion should predict the initiation of a 'global' defect, defined here as the crack network and estimate its characteristics: crack density, depth...A propagation law should then predict the evolution of the characteristics of the crack networks with growing number of cycles.

\section{Acknowledgements}

The authors would like to thank Prof. H. Maitournam (LMS, CNRS 7649 - Ecole Polytechnique) and Dr. E. Charkaluk (LML, CNRS UMR 8107 - Lille) for fruitful discussions regarding the triaxiality factor in the fatigue criterion and M. Payen (IRSN - Institut de Radioprotection et de Sureté Nucléaire) for his particular support of this project.

\section{REFERENCES}

1 Marsh, D. J. (1981) A thermal-shock fatigue study of type-304 and type-316 stainless-steels. Fatigue Eng. Mater. Struct. 4, 179-195.

2 Amiable, S., Chapuliot, S., Constantinescu, A. and Fissolo, A. (2006) A computational lifetime prediction of a thermal shock experiment. Part II : discussion on difference fatigue criteria. Fatigue Fract. Eng. Mater. Struct. doi: 10.1111/j.1460-2695. 2006.0983.x.

3 RCC-MR French code, 1993.

4 Charkaluk, E. and Constantinescu, A. (2000) An energetic approach in thermomechanical fatigue for silicon molybdenum cast iron. Mater. High Temp. 17, 373-380.

5 Constantinescu, A., Charkaluk, E., Lederer, G. and Verger, L. (2004) A computational approach to thermomechanical fatigue. Inter. F. Fatigue 26, 805-818.

6 Sermage, J.P., Lemaitre, J. and Desmorat, R. (2000) Multiaxial creep-fatigue under anisothermal conditions. Fatigue Fract. Eng. Mater. Struct. 23, 241-253.

7 Fissolo, A., Marini, B., Nais, G. and Wident, P. (1996) Thermal fatigue behaviour for a 3161 type steel. 7 . Nucl. Mater. 23, 156-161.

8 Maillot, V. Amorçage et Propagation de Réseaux de Fissures de Fatigue Thermique dans un Acier Inoxydable Austenitique de Type X2 CrNi18-09 (AISI 304L). (2003), Ecole Centrale de Lille.

9 Mottot, $M$. Etude du comportement en fatigue oligocyclique à 165 et $320^{\circ} \mathrm{C}$ du 3041 (tole t112) pour de faibles niveaux de deformation $\left(\delta \epsilon_{\mathrm{t}} \leq 1 \%\right)$. (2001) TN SRMA 2001-2403, CEA, Janvier.

10 Lemaitre, J. and Chaboche, J.L. (1988) Mécanique des Matériaux Solides. Dunod.

11 Krempl, E. and Khan, F. (2003) Rate (time)-dependant deformation behavior: an overview of some properties of metal and solid polymers. Inter. F. Plast. 19, 1069-1095 
12 Benallal, A. and Marquis, D. (1987) Constitutive equations for nonproportional cyclic elastoviscoplasticity. F. Eng. Mater. Technol. 109 326-336.

13 Skelton, R.P. (1991) Energy criterion for high temperature low cycle fatigue failure. Mater. Sci. Technol. 7, 427-439.

14 Constantinescu, A., Charkaluk, E., and Verger, L. (2001) A macroscopic crack initiation criterion in multiaxial thermomechanical fatigue. In 6 th International Conference on Biaxial Multiaxial Fatigue \& Fracture, Lisbon, Portugal.
15 Thomas, J.J., Verger, L., Bignonnet, A. and Charkaluk, E. (2004) Thermomechanical design in the automotive industry. Fatigue Fract. Eng. Mater. Struct. 27, 887-895.

16 Documentation Cast 3 M. http://www-cast3m.cea.fr.

17 Documentation Mathematica. http://www.wolfram.com.

18 Dang Van, K. and Papadopoulos, I. (1999) High Cycle Metal Fatigue. Springer Wien: New York.

19 Itoh, T., Sakane, M., Ohnami, M. and Socie, D. (1995) Nonproportional low cycle fatigue criterion for type 304 stainless steel. 7. Eng. Mater. Technol. 117 285-292. 